\title{
Design of Solar Automatic Tracking Control System Based on STC89C52 Zhaoyang Qiao ${ }^{1, \text { a }}$ \\ ${ }^{1}$ School of Computer Science, Baicheng Normal College, Baicheng,137000 China \\ a email: qzy1969@126.com
}

Keywords: Automatic Tracking; Solar Energy; STC89C52; Avoidance

\begin{abstract}
Aiming at the low generating efficiency of the current solar energy generating system, solar energy maximum power point tracking control system based on STC89C52 is designed and made. The photoelectric detection and tracking is adopted as the control mode in the system. By using stepping motor as driving mechanism, comprehensive trace of the sun is realized by controlling the movement of tracking mechanism in the horizontal and pitching directions. Based on this, real-time detection of wind velocity and change of wind direction is realized by wind velocity and direction transducer which is equipped on the tracking mechanism, which makes the system automatically avoid the typhoon above level 8 to weaken the damage to panel by the storm. The experiment results of model machine indicate that the system has reliable performance which can satisfy the need of auto-solar track, it can also make the panel orient towards east again after darkness to realize daily circular run and it is of relatively high practical value.
\end{abstract}

\section{Introduction}

With the continuous development of the society, the human being has a greater demand for energy sources[1]. At present, most of the energy sources we use are got from nonrenewable resources such as coal, petroleum, natural gas, etc., which are limited. Moreover, during the combustion process there will be plenty of greenhouse gases which bring environmental problems [2][3]. This fact makes people pay more attention to renewable resources among which solar energy has already been used for a certain period. In the common solar energy generating control system, panel stationary[4] solution is used. At the same time, solar energy[5][6][7] has the drawbacks including low density, intermittency and constant change of space distribution which lead to the low use ratio of solar energy for the present solar energy equipments. A maximum power automatic tracking control system moving with the sun from the practical perspective is designed in this paper, which effectively increases the utilization efficiency of solar energy.

\section{Hardware design of the system}

The whole hardware of the system is composed of control segment and driving segment. The control segment mainly includes STC89C52 main control panel, light intensity detection module, liquid crystal display, wind velocity and direction detection module and angle detection module. The driving segment mainly includes mechanism support, solar panel, worm-gear pair, stepping motor and its actuator. The driving structure of the system is shown in picture 2 and the hardware structure diagram of the whole system is shown in Fig. 1.

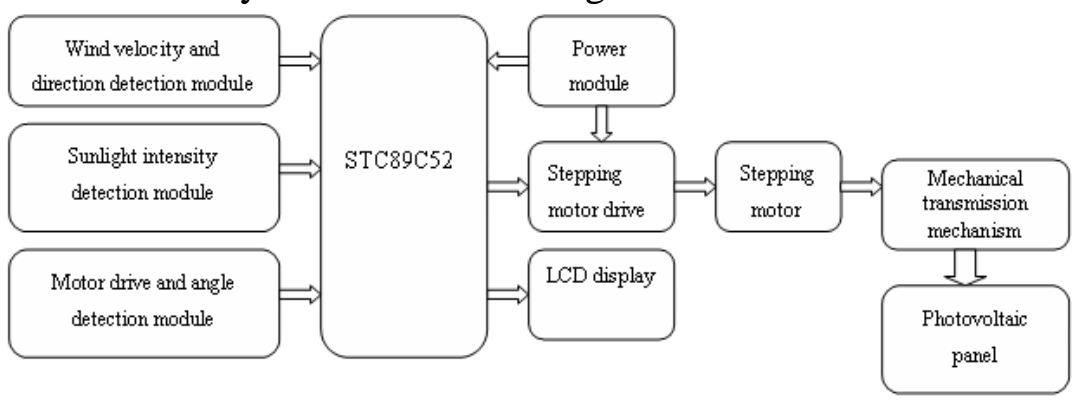

Fig. 1Hardware structure diagram of the system 


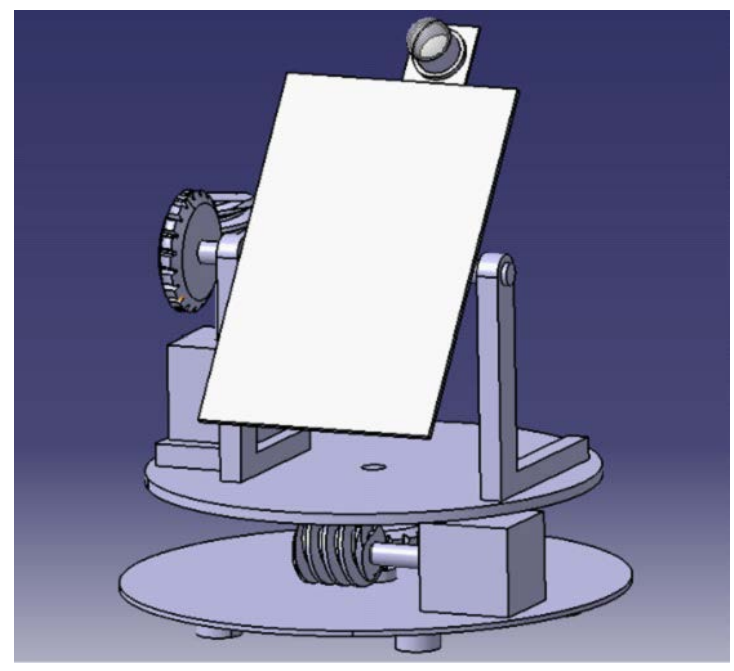

Fig. 2 3D diagram of drive structureof the system

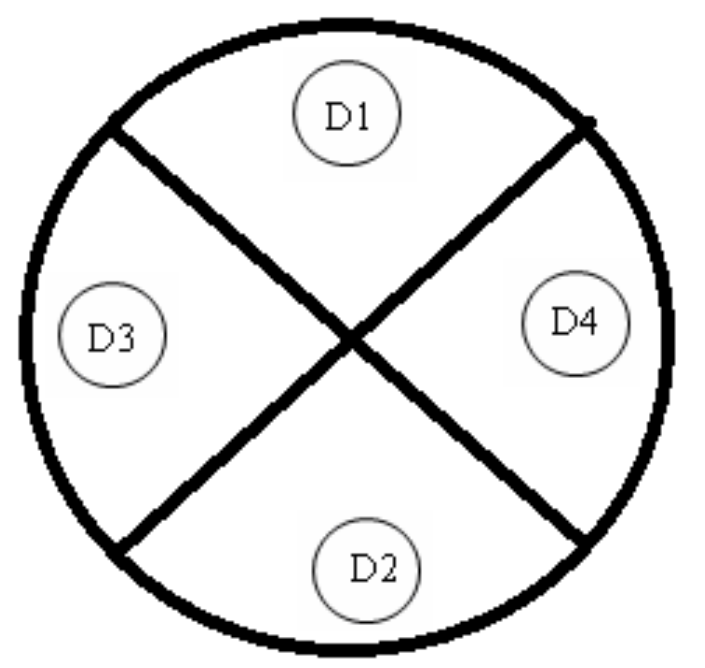

Fig. 3Top view of photoelectric detection module

Circuit design of light intensity detection. Photoelectric detection circuit is mainly to detect the change of solar elevating angle and azimuth angle in order to realize the tracking and orientation of the sun. Top view of photoelectric detection module is shown in Fig. 3. Four totally same phototriodes are put in a light intensity collection plate of the cross type. The collection plate is divided into upper area, lower area, right area and left area by the cross. Phototriodes D1 and D2 are used to detect elevating angle of the sun while phototriodes D3 and D4 are used to accurately detect the azimuth angle of the sun. The solar panel will have the fine adjustment through the measurement of elevating angle and azimuth angle to make it directly face the sun. Schematic circuit diagram of photoelectric detection module is shown in Fig. 4. 


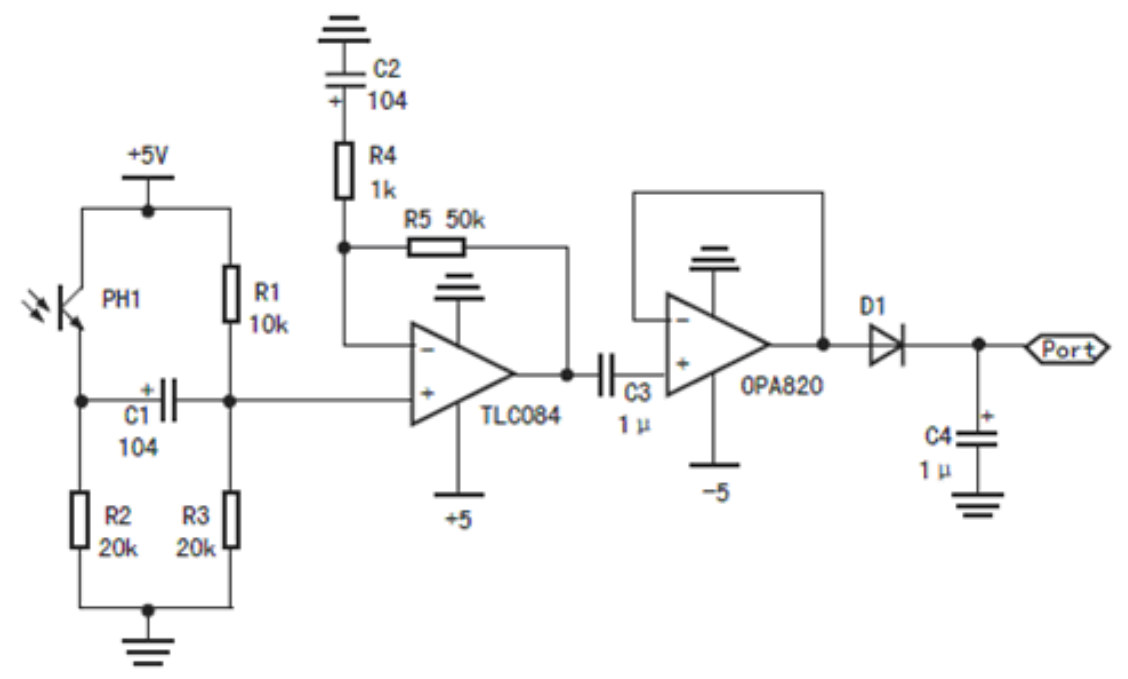

Fig. 4Schematic circuit diagram of photoelectric detection module

Design of automatic avoidance of the system.Considering that panels are in the hostile outdoor environment all year round, it is designed to prolong the life of the panels. Real-time detection of wind velocity and change of wind direction is realized by wind velocity and direction transducer. When wind velocity has reached the certain value, master control unit will receive an interrupt signal from wind velocity through which master control module will control the motor action and make the plan position of solar panels be parallel with the wind direction in order to avoid the damage to solar panels by too high wind velocity. Schematic circuit diagram of system automatic avoidance is shown in Fig. 5

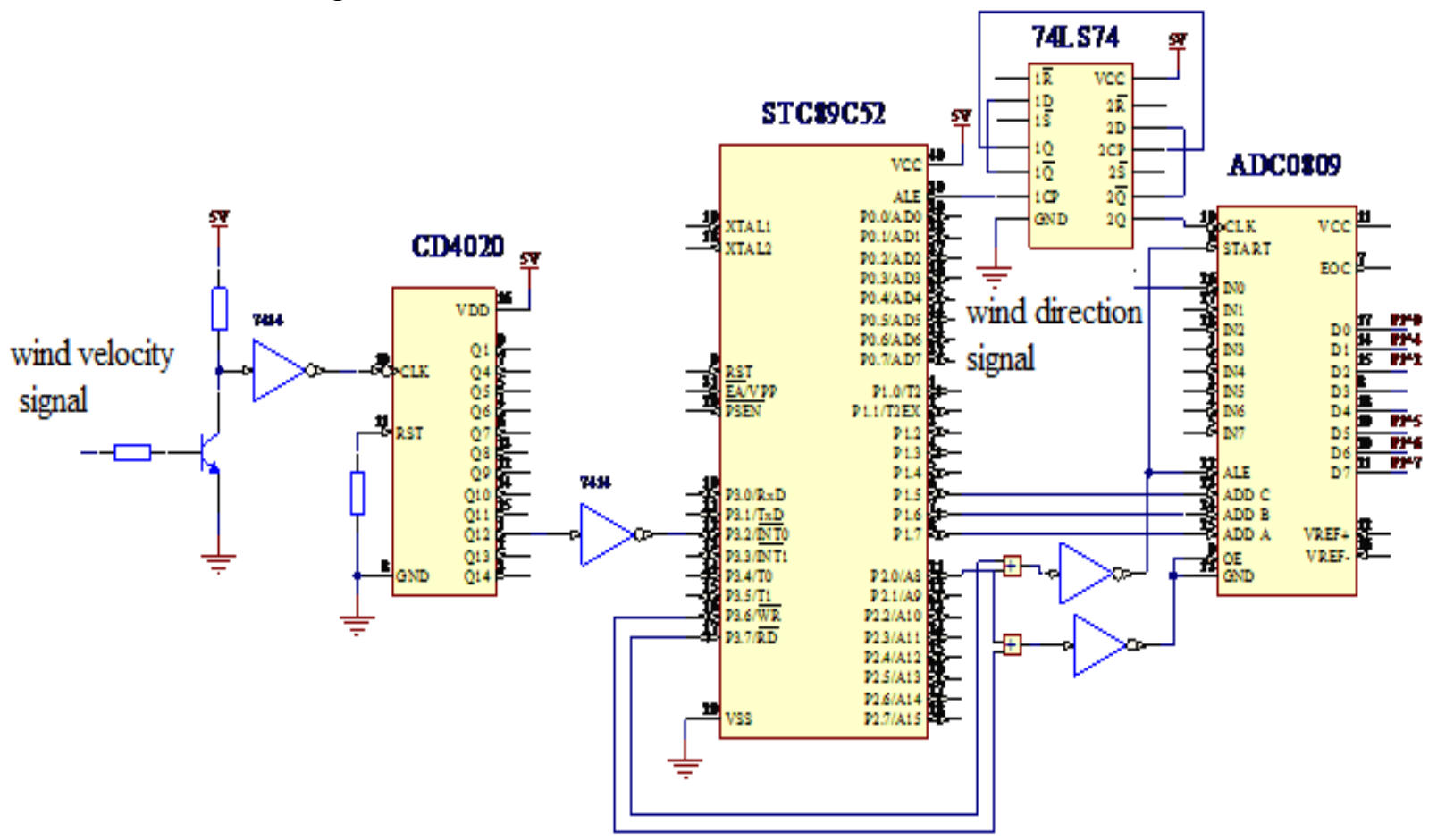

Fig. 5Schematic circuit diagram of system automatic avoidance

\section{Software design}

C programming is the major part in the software design of system maximum power point tracking control, In order to save internal storage and ensure strong adaptability, structured programming is applied and common programs is complied as subprograms (for example, wind power interruption subprogram, light intensity detection subprogram, motor drive subprogram, etc). 
then, combine these subprograms by certain rules and the application program finishing certain task can be acquired. The flow diagrams of main program and automatic avoidance program of the system are shown in Fig. 6 and Fig. 7.

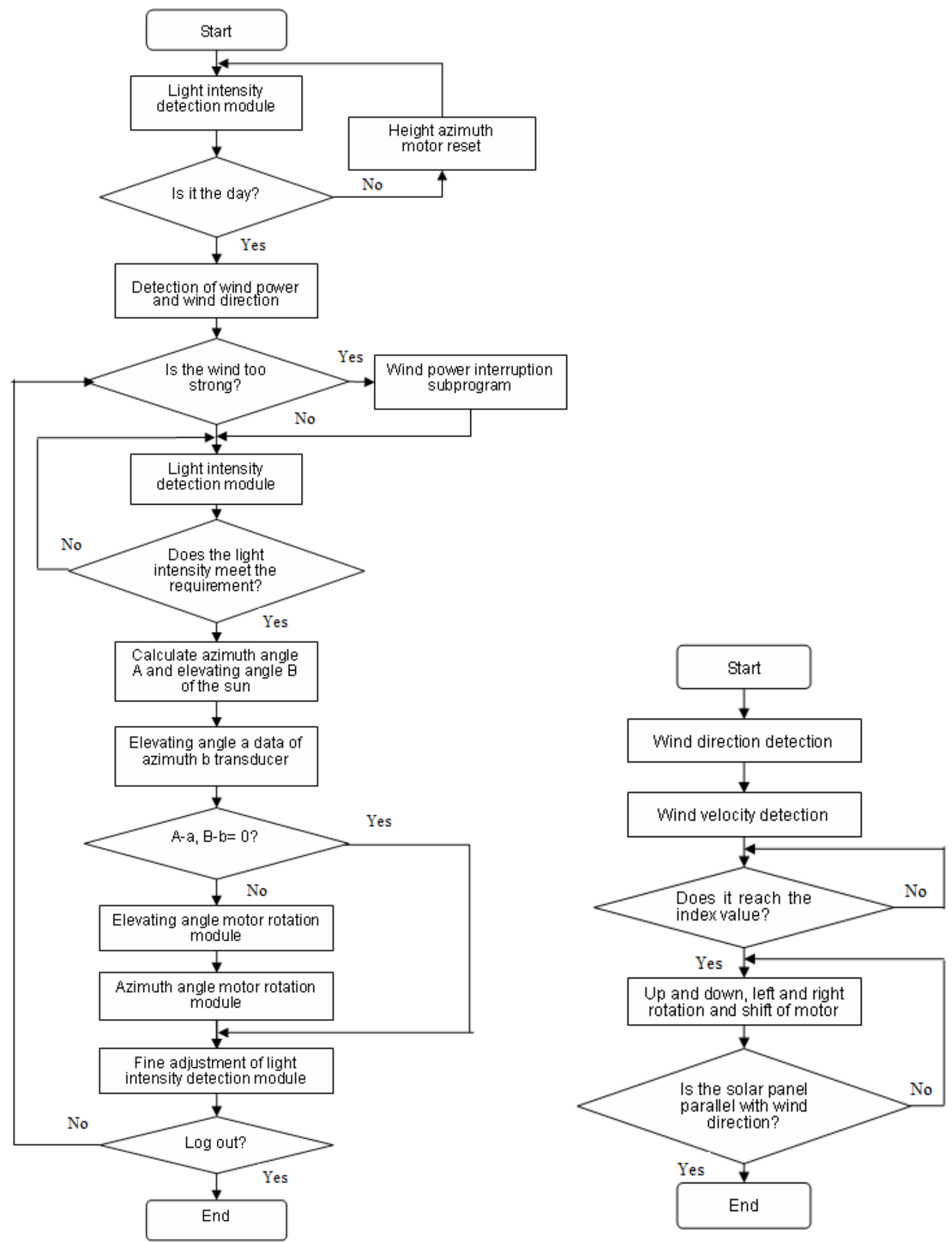

Fig. 6 Flow diagram of system main program

Fig. 7 Flow diagram of automatic avoidance program 


\section{Experimental Results}

All-day sunlight experiment is done outdoors. Two solar panels with the same standard are used in the experiment. One is adjusted to the best angle and then fixed while the designed solar energy maximum power automatic tracking system is used in the other one. From 7:00 to 17:00, test lighting intensity of solar panels respectively every an hour. The correlation curve is shown in picture 8 from which it can be seen that compared with fixed installation of solar panels, solar irradiation of solar panels which uses solar energy automatic tracking system is increased and the use ratio of solar energy is also improved.

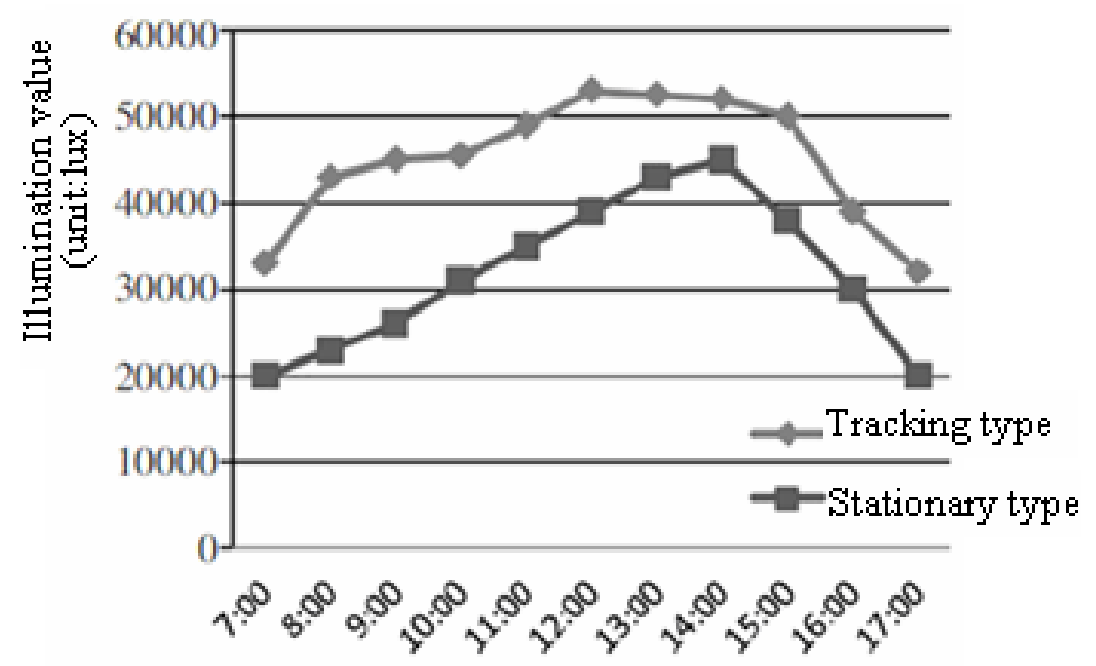

Fig. 8 Correlation curve of light intensity of tracking and stationary solar panels

\section{Conclusion}

In order to improve the sun tracking precision and increase use ratio of solar energy, a solar automatic tracking control system based on single chip is designed. Microprocessor chip STC89C52 is used as controller in this system. The comprehensive tracking of the sun is realized by program controlling of horizontal and pitching directions of the tracking mechanism. After darkness, it can make the panel face east again and daily circular running is realized. Besides, the system has the function of automatic avoidance to weaken the damage of storm to panels and prolong its life. The design is verified by the physical model. The system is operated smoothly with small tracking accuracy error. It has certain significance in realizing large-scale solar power generation and research and popularization of solar automatic tracking control of solar aircraft.

\section{References}

[1]Ding Wei: Research and Implementation of automatic tracking control system of solar power generation(Nanjing University of Aeronautics and Astronautics Publications, Jiang Su2010).

[2]Reda I,Andreas A: Solar position algorithm for solar radiation applications. Solar Energy. Vol.76(5)(2004), p. 577-589

[3]Zhang Dongyu: Single-dimension dragging and double-dimensions tracing intelligent solar tracking system.Control \& Automation.Vol.22(16) (2006), p.31-35

[4]Xiang Ping,Bi Yuqing,Cheng Jianmin: Design of sun tracking control system based on ARM. Computer Measurement \& Control. Vol.17(6)(2009) , p. 1102-1105

[5]Geng Xin,Liu Zhongda: Design of intelligent automatic sun tracking system based on PLC .Industrial Instrumentation \& Automation. Vol.13(3)(2010), p. 30-33 
[6]Wang Bin,Zhang Xun: Automatic real-time solar tracking system. Astronomical Research \& Technology. Vol.8(1)(2011), p. 52-57

[7]Roth P,Georgiev A: Design and construction of a system for sun tracking. Renewable Energy. Vol.29 (3)(2004), p. 393-402 